\title{
FRATURA DO QUADRIL: AVALIAÇÃO PÓS-OPERATÓRIA DO RESULTADO CLÍNICO E FUNCIONAL
}

\author{
HIP FRACTURE: POST-OPERATIVE EVALUATION \\ OF CLINICAL AND FUNCTIONAL OUTCOMES
}

Marcelo Teodoro Ezequiel Guerra' ${ }^{1}$, Thomas Alexandre Thober² ${ }^{2}$ André Vicente Bigolin ${ }^{3}$, Marcos Paulo de Souza ${ }^{4}$, Simone Echeveste 5 .

\section{RESUMO}

Objetivo: Avaliar o resultado clínico e funcional dos pacientes submetidos à cirurgia por fratura do quadril relacionando com o escore ASA e com tempo para o tratamento cirúrgico definitivo. Métodos: No período de um ano foram operados 154 pacientes com 65 anos ou mais, com fratura do quadril. Dados sobre o escore pré-operatório ASA e sobre o tempo de espera para a cirurgia foram obtidos. Dois anos após a cirurgia, foi aplicado o questionário Escore de Recuperação Funcional de Zuckerman (ERF) como examinadorda capacidade funcional atual dos pacientes. Resultados: A mortalidade no primeiro ano pós-operatório apresentou diferença entre pacientes com ASA 3 ou 4 em relação aos classificados como ASA 1 ou 2 (dado significante $\mathrm{p}<0,05$ ). A mortalidade até o final do segundo ano pós-operatório foi significantemente maior $(\mathrm{p}<0,05)$ no grupo ASA 3 ou 4. O escore pré-operatório ASA não apresentou relação significante com a capacidade funcional atual dos pacientes (dado significante $\mathrm{p}<0,05$ ). Não houve diferença significante entre $\mathrm{o}$ grupo operado em menos de 48 horas da admissão do grupo operado após 48 horas, em relação à mortalidade e à capacidade funcional atual (dado significante $\mathrm{p}<0,05$ ). $\mathrm{O}$ grupo de 80 anos ou mais apresentou mortalidade significantemente maior $(\mathrm{p}<0,05)$ do que o grupo de 65 a 79 anos até o final do segundo ano pós-operatório. Conclusão: O escore pré-operatório ASA e a idade maior de 80 anos podem ser considerados fatores associados à maior mortalidade após dois anos de pós-operatório por fratura do quadril. Isoladamente, o tempo para o tratamento cirúrgico não foi significante.

Descritores - Fraturas do Quadril; Período Pós-Operatório; Saúde do Idoso

\section{ABSTRACT}

Objective: To evaluate the clinical and functional outcomes of patients submitted to surgery for hip fracture through the ASA score and time for definitive surgical treatment. Method: During one year, 154 patients with hip fractures, aged 65 years and over, were operated on. Data from the pre-operative ASA score and time awaiting operation were obtained. Two years after the operation, Zuckerman's Functional Recovery Score (FRS) questionnaire was used to assess the current functional capacity of the patients. Results: Mortality in the first postoperative year was different between patients with an ASA 3 or 4 compared to those classified as ASA 1 or 2 group (significant data $p$ <0.05). Mortality up to the end of the second postoperative year was significantly higher $(p<0.05)$ in the ASA 3 or 4 group. The preoperative ASA score didn't demonstrate a significant relationship to the current functional capacity of the patients $(p>0.05)$. There was no significant difference between the group operated within 48 hours of admission and the group operated after 48 hours in relation to mortality or current functional capacity ( $p>0.05)$. The group aged 80 and over showed significantly higher mortality than the group aged 65 to 79 years through the end of the second post-operative year ( $p>0.05)$. Conclusion: A preoperative ASA score and an age of 80 or over may be considered factors associated with higher mortality two years post-operatively after hip fracture. In isolation, time awaiting surgery was not significant.

Keywords - Hip Fractures; Postoperative Period; Health of the Elderly

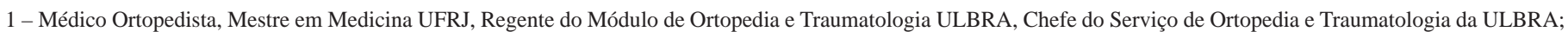
Titular da SBOT e SBTO; International Affiliate of AAOS.

2 - Médico Residente de Ortopedia e Traumatologia do Hospital Cristo Redentor, Porto Alegre, RS.

3 - Acadêmico do Curso de Medicina da ULBRA, Canoas, RS.

4 - Médico Ortopedista, Membro do Grupo de Trauma Ortopédico da ULBRA, Canoas, RS.

5 - Mestre em Estatística e Professora de Estatística da ULBRA, Canoas, RS.

Trabalho realizado na Universidade Luterana do Brasil - ULBRA, Canoas, RS.

Correspondência: Rua Nilo Peçanha, 450, apto. 901. Bairro Petrópolis CEP 90470-000 - Porto Alegre, RS.

E-mail: mguerraz@terra.com.br

Trabalho recebido para publicação: 31/12/09, aceito para publicação: 13/07/10. 


\section{INTRODUÇÃO}

As fraturas do quadril incluem as fraturas intertrocantéricas e as fraturas do colo do fêmur, e constituem um grande problema médico devido a suas altas taxas de morbidez e mortalidade. A incidência de fraturas do quadril aumenta com a idade, dobrando a cada 10 anos depois dos 50 anos $^{(1-5)}$. Esse aumento é consideravelmente maior depois da menopausa nas mulheres e depois dos 70 anos nos homens ${ }^{(1-4,6-8)}$. Nos Estados Unidos ocorrem aproximadamente 250.000 casos por ano, com um custo anual de cerca de 14 bilhões de dólares. A incidência geral de mortalidade no primeiro ano pós-fratura varia de 10 a 30\% ${ }^{(1,4-6,9,10)}$. Inúmeros fatores estão relacionados com um risco aumentado de mortalidade no pós-operatório e que influenciam também no potencial de reabilitação a longo $\operatorname{prazo}^{(11-20)}$.

A avaliação pré-operatória e o seguimento pós-operatório desse grupo de pacientes é fundamental para se atingir o resultado clínico e funcional esperado a longo prazo $^{(21-23)}$. Um dos métodos para a avaliação é o escore pré-operatório da Sociedade Americana de Anestesiologistas (ASA), que tem sido comprovado como um importante fator preditor de mortalidade no primeiro ano pós-operatório por fratura do quadril ${ }^{(24-27)}$. Outro método é o Escore de Recuperação Funcional (ERF) desenvolvido por Zuckerman em 1999, que demonstrou ser confiável para a avaliação do resultado funcional pós-fratura $^{(10,28)}$.

Este estudo tem como objetivo avaliar variáveis como idade, escore pré-operatório ASA e tempo de atraso cirúrgico, em relação à mortalidade e ao resultado funcional em pós-operatório tardio de fratura de quadril em pacientes com 65 anos de idade ou mais.

\section{MATERIAIS E MÉTODOS}

Os pacientes foram operados por fratura do quadril no Hospital Independência de Porto Alegre no período entre janeiro de 2004 e dezembro de 2005. Foram incluídos no estudo todos os pacientes com 65 anos ou mais no momento da cirurgia, que sofreram fratura do quadril (fraturas intertrocantéricas ou do colo do fêmur) de origem não patológica, em qualquer estado cognitivo e funcional prévio, com a devida indicação clínica e cirúrgica e independentemente do tipo e da abordagem cirúrgica. Foram excluídos os pacientes com fratura subtrocantérica e aqueles em que não havia nenhum número telefônico registrado no prontuário para contato posterior.
Os dados referentes ao nome do paciente, data e tipo de cirurgia e cirurgião responsável foram coletados. Idade, número telefônico (incluindo de familiares próximos ou responsáveis), tempo da admissão até a realização da cirurgia e o escore da avaliação préoperatória ASA descrito pelo anestesista responsável foram obtidos dos prontuários.

O questionário do Escore de Recuperação Funcional foi aplicado em um único momento (junho de 2007), mais de um ano e meio após a cirurgia, a todos os pacientes em que foi possível o contato por telefone (média de dois anos e dois meses após a cirurgia, variando de um ano e seis meses a três anos e cinco meses). Familiares próximos ou responsáveis também responderam o questionário nos casos em que não foi possível o contato direto com o paciente. Também foi questionado sobre a causa e a data dos possíveis óbitos.

Para fins de análise estatística e conforme estudos anteriores $^{(9,27-29)}$, os pacientes foram separados em grupos, de acordo com os fatores a serem analisados. Em relação ao escore pré-operatório ASA, estratificamos em ASA 1 ou 2 e ASA 3 ou 4. Em relação ao tempo de atraso cirúrgico, estratificamos no grupo operado em menos de 48 horas da admissão e no grupo operado após 48 horas. Dividimos também por faixas etárias, sendo um grupo formado pelos pacientes de 65 a 79 anos e o outro pelos de 80 anos ou mais. Em relação ao resultado funcional, estratificamos os pacientes em ERF 80 a 100, 60 a 79 e menor do que 60. A análise estatística foi realizada pelo teste não paramétrico de Mann Whitney utilizando 5\% de nível de significância para verificar se existiu diferença significativa entre os grupos em relação à mortalidade no primeiro e segundo ano pós-operatório e em relação à capacidade funcional atual medida pelo ERF. As análises foram realizadas com auxílio do software SPSS versão 10 .

\section{RESULTADOS}

No período de dois anos do estudo foram operados 207 pacientes. Cinquenta e três não apresentaram critérios para inclusão, restando 154 pacientes. Dos 154, apenas 79 pacientes participaram do estudo. Setenta e cinco pacientes foram perdidos do estudo por não ter sido possível o contato por telefone. A idade média foi de 78,6 anos (65 a 93 anos). Tivemos 41 pacientes (51,9\%) entre 65 e 79 anos e 38 (48,1\%) com 80 anos ou mais. Sessenta pacientes (75,9\%) eram do sexo feminino e $19(24,1 \%)$ do sexo masculino. Entre as mulheres, 
cinco morreram no primeiro ano pós-operatório e sete no segundo. Entre os homens, quatro morreram no primeiro pós-operatório e dois no segundo. Houve um total de 18 óbitos (22,8\%), sendo nove no primeiro e nove no segundo ano pós-operatório. As causas das mortes foram basicamente: complicações respiratórias, acidente vascular cerebral e infarto agudo do miocárdio.

O grupo com escore pré-operatório ASA 1 ou 2 teve 43 pacientes $(54,4 \%)$. Desses, três morreram no primeiro ano pós-operatório e três no segundo. O grupo com escore 3 ou 4 teve 36 pacientes (45,6\%). Desses, seis morreram no primeiro ano e seis no segundo. Os dados dos pacientes são apresentados na Tabela 1. O grupo

Tabela 1 - Perfil dos pacientes avaliados no estudo quanto as variáveis avaliadas $(n=79)$.

\begin{tabular}{|c|c|c|}
\hline Idade & & \\
\hline 65 a 79 anos & 41 & $51,90 \%$ \\
\hline 80 anos ou mais & 38 & $48,10 \%$ \\
\hline \multicolumn{3}{|l|}{ Sexo } \\
\hline Feminino & 60 & $75,90 \%$ \\
\hline Masculino & 19 & $24,10 \%$ \\
\hline \multicolumn{3}{|l|}{ Escore ASA } \\
\hline 1 ou 2 & 43 & $54,40 \%$ \\
\hline 3 ou 4 & 36 & $45,60 \%$ \\
\hline \multicolumn{3}{|l|}{ Tempo até cirurgia } \\
\hline Mais de $48 \mathrm{~h}$ & 47 & $59,50 \%$ \\
\hline Menos de 48h & 32 & $40,50 \%$ \\
\hline \multicolumn{3}{|l|}{ Óbito } \\
\hline Não & 61 & $77,20 \%$ \\
\hline $1^{0}$ ano pós-operatório & 9 & $11,40 \%$ \\
\hline $2^{\circ}$ ano pós-operatório & 9 & $11,40 \%$ \\
\hline \multicolumn{3}{|l|}{ Capacidade Funcional Atual } \\
\hline \multicolumn{3}{|l|}{ Atividades básicas do dia a dia (BADL) } \\
\hline Dependente em pelo menos uma & 28 & $45,90 \%$ \\
\hline Independente em todas & 33 & $54,10 \%$ \\
\hline \multicolumn{3}{|l|}{ Atividades instrumentais do dia a dia (IADL) } \\
\hline Dependente em pelo menos duas & 33 & $54,10 \%$ \\
\hline Dependente em apenas uma ou nenhuma & 28 & $45,90 \%$ \\
\hline \multicolumn{3}{|l|}{ Capacidade de deambulação } \\
\hline Independência plena & 21 & $34,40 \%$ \\
\hline $\begin{array}{l}\text { Deambulador com suporte: bengala/andador/ } \\
\text { acompanhante }\end{array}$ & 20 & $32,80 \%$ \\
\hline Deambula apenas em casa & 12 & $19,70 \%$ \\
\hline Não deambula & 8 & $13,10 \%$ \\
\hline
\end{tabular}

operado em menos de 48 horas da admissão teve 32 pacientes (40,5\%). Desses, dois morreram no primeiro ano pós-operatório e quatro no segundo. O grupo operado após 48 horas teve 47 pacientes (59,5\%). Desses, sete morreram no primeiro ano e cinco no segundo.

Em relação à capacidade funcional atual dos pacientes, o ERF medido em média dois anos após a cirurgia foi o seguinte: escore 80 a 100, 30 pacientes (38\%), escore 60 a 79, $10(12,7 \%)$ e escore menor do que 60, 21 (26,6\%). O grupo dependente em pelo menos uma atividade básica de vida diária (ABVD) teve 28 pacientes $(45,9 \%)$ sendo o restante independente em todas as atividades. O grupo dependente em pelo menos duas atividades instrumentais de vida diária (IAVD) teve 33 pacientes (54,1\%), sendo o restante dependente em apenas uma ou independente em todas as atividades. Vinte e um pacientes $(34,4 \%)$ apresentaram independência plena para deambular, tanto em casa como fora. Vinte pacientes (32,8\%) necessitam de suporte para deambular fora (bengala, andador, acompanhante). Doze pacientes $(19,7 \%)$ conseguem somente deambular dentro de casa e oito pacientes $(13,1 \%)$ não deambulam.

O grupo de 80 anos ou mais apresentou mortalidade maior ( $\mathrm{p}<0,05)$ do que o grupo de 65 a 79 anos até o final do segundo ano pós-operatório. Quando avaliada apenas a mortalidade no primeiro ano pós-operatório, não foi encontrada diferença entre os grupos ( $>$ > 0,05). Também não foi encontrada diferença em relação à capacidade funcional atual entre os grupos etários ( $\mathrm{p}>$ $0,05)$. Óbito entre os homens foi proporcionalmente maior do que entre as mulheres, mas a diferença não foi estatisticamente significante $(p>0,05)$. Em relação à capacidade funcional atual, não houve diferença entre homens e mulheres $(\mathrm{p}>0,05)$ A mortalidade até o final do segundo ano pós-operatório foi maior no grupo ASA 3 ou $4(p<0,05)$ em relação ao grupo ASA 1 ou 2. A mortalidade no primeiro ano pós-operatório isoladamente não foi maior no grupo ASA 3 ou 4 em relação ao grupo ASA 1 ou 2 (p > 0,05). O escore pré-operatório ASA não mostrou relação significante $(p>0,05)$ com o resultado funcional atual dos pacientes. O grupo operado em menos de 48 horas da admissão e o grupo operado após 48 horas não diferiram em relação à mortalidade e à capacidade funcional atual $(\mathrm{p}>0,05)$.

\section{DISCUSSÃO}

O grande viés do nosso estudo, que comprometeu a análise dos dados, foi o tamanho da amostra. Prati- 
camente metade dos pacientes não pôde ser contatada por telefone. Dessa forma, não foi possível confirmar achados de outros estudos. O estudo também é retrospectivo, sendo que o escore ASA, em alguns casos, teve de ser calculado por nós. A aplicação do questionário foi realizada através de contato por telefone, um método discutível apesar de já ter sido demonstrada a sua confiabilidade por Zuckerman et $a l^{(10,28)} \mathrm{e}$ Petrella et $a l^{(30)}$.

O escore de avaliação pré-operatória da Sociedade Americana de Anestesiologistas (ASA) tem sido comprovado como um importante fator preditor de mortalidade no primeiro ano pós-operatório por fratura do quadril ${ }^{(24-27)}$. Ele é empregado quase universalmente há mais de 30 anos, sendo uma tentativa de padronizar a avaliação clínica e estimar o risco peri-operatório ${ }^{(31,32)}$. Em nosso estudo, seis pacientes do grupo ASA 3 ou 4 e três pacientes do grupo ASA 1 ou 2 foram ao óbito no primeiro ano pós-operatório. A diferença entre os óbitos não foi estatisticamente significante. Entretanto, no estudo de Michel et $a l^{(27)}$, foi demonstrado que o grupo que apresentava o escore ASA 3 ou 4 teve um risco quase nove vezes maior de morte no primeiro ano pós-operatório do que o grupo ASA 1 ou 2 ( $\mathrm{p}<0,001$ ), principalmente nos primeiros três meses da fratura ou da cirurgia. Nesse estudo, não houve relação significante do escore pré-operatório ASA com a recuperação funcional a longo prazo. Em nosso estudo, dos nove pacientes que morreram no primeiro ano, quatro morreram nos primeiros três meses pós-cirurgia. Dzupa et $a^{(26)}$ encontraram alto risco de mortalidade relacionado ao sexo masculino, maiores de 80 anos e ASA 4, principalmente nos primeiros três meses da cirurgia. Da mesma forma, Hasegawa et $a^{(25)}$ demonstraram que o escore ASA 3 ou 4, além de outros quatro fatores (gênero, idade avançada, demência e institucionalização dos pacientes), estão intimamente relacionados com um maior risco de mortalidade no pós-operatório por fratura do quadril. Richmond et al ${ }^{(33)}$ também relataram que, entre os paciente com menos de 85 anos, aqueles com classificação ASA 3 ou 4 tiveram uma mortalidade significativamente excessiva $(\mathrm{p}<0,05)$ durante $\mathrm{o}$ acompanhamento de dois anos após a fratura. No estudo de Zuckerman et $a l^{(10,28)}$, 69 pacientes $(11,6 \%)$ morreram durante o primeiro ano pós-operatório. Nakano ${ }^{(9)}$ encontrou, no Japão, mortalidade de $10 \%$ no primeiro ano pós-operatório $(\mathrm{n}=10.992)$. Em nosso estudo, o valor foi bastante aproximado do encontrado nesses estudos (11,2\%). Zuckerman et al ${ }^{(10,28)}$ demonstraram que os pacientes com um escore pré-operatório ASA 3 ou 4 tinham o ERF mais baixo anteriormente à fratura do que aqueles com ASA 1 ou 2 ( $p<0,001)$. Em nosso estudo, encontramos um número aparentemente bem maior de pacientes com melhor capacidade funcional atual que apresentavam escore ASA 1 ou 2 ( $\mathrm{n}=22$ ). Porém, quando feita a análise estatística, essa diferença não foi significante.

Em relação ao tempo de atraso cirúrgico, Hamlet et $a^{(34)}$ demonstraram que pacientes operados dentro das primeiras 24 horas da admissão tiveram mortalidade menor do que aqueles operados depois de 24 horas, independentemente do escore pré-operatório ASA. Da mesma forma, Casaletto e Gatt ${ }^{(20)}$ evidenciaram que a sobrevivência em um ano é melhor quando os pacientes estão clinicamente prontos para a cirurgia e operados no mesmo dia da admissão. Esta vantagem da sobrevivência é maior nos pacientes acima de 80 anos. McGuire et $a l^{(35)}$ também encontraram que o atraso cirúrgico de dois ou mais dias aumentou significantemente a mortalidade em um mês. Porém, nos estudo mais recentes como o de Bergeron et al $^{(29)}$, demonstraram que o atraso cirúrgico não tem relação com resultados adversos quando a cirurgia é atrasada para permitir o tratamento de comorbidades. O atraso para a cirurgia está associado com uma permanência mais longa no hospital. Da mesma forma, McLeod et al ${ }^{(36)}$ demonstraram que fatores não relacionados ao paciente e ao processo, incluindo atraso cirúrgico, tipo de cirurgia e tipo de anestésico, tiveram impacto mínimo na mortalidade em um ano. Nenhum determinante principal para o tempo de permanência no hospital foi identificado. O estado de saúde foi o determinante principal para o atraso cirúrgico. E também, Williams e Jester ${ }^{(37)}$ mostraram não haver nenhuma relação entre o atraso cirúrgico e a mortalidade pós-operatória $(\mathrm{p}<0,05)$ quando todas as outras variáveis independentes foram controladas. Segundo o seu estudo, a disfunção cognitiva e a mobilidade reduzida antes da fratura foram bons indicadores prognósticos de mortalidade aumentada no primeiro ano pós-operatório. Em nosso estudo, o tempo de atraso cirúrgico maior do que 48 horas não mostrou estar relacionado com uma mortalidade mais elevada no primeiro ano pós-operatório nem com a capacidade funcional atual ( $>$ > 0,05).

A imensa maioria dos instrumentos de avaliação pósfratura do quadril limita-se apenas ao estado geral de saúde e ao bem-estar do paciente ${ }^{(38-40)}$. São geralmente 
complexos e difíceis de serem aplicados em idosos. Basicamente, enfatizam o alívio da dor, um sintoma que normalmente não está presente anteriormente à fratura do quadril. Além do mais, os resultados avaliados são comumente baseados naquilo que os planos de saúde consideram mais importante: consolidação da fratura, alinhamento e infecção( ${ }^{(28)}$. O Escore de Recuperação Funcional de Zuckerman et al ${ }^{(10,28)}$ é um questionário que pode ser aplicado tanto em consultas ambulatoriais quanto por telefone. Consiste em 11 perguntas relacionadas às atividades de vida diária: quatro relacionadas à independência nas atividades básicas, seis relacionadas às atividades instrumentais e uma relacionada à mobilidade. Escores pré-fratura utilizando o ERF demonstraram que o escore apresenta valor preditivo para mortalidade, institucionalização dos pacientes e re-hospitalização no primeiro ano após a fratura. No estudo de Zuckerman et $a l^{(10,28)}$, que validaram o Escore de Recuperação Funcional, todos os pacientes foram identificados após a fratura do quadril na admissão e prospectivamente seguidos. $\mathrm{O}$ dado sobre a capacidade funcional prévia à fratura foi coletado no momento da admissão. Durante o seguimento, as informações foram obtidas em três, seis e 12 meses após a cirurgia, através de entrevistas diretas com o paciente utilizando-se sempre o questionário do Escore de Recuperação Funcional.

O nosso estudo não teve valor preditivo como o de Zuckerman et al ${ }^{(10,28)}$, pelo fato de o questionário sobre recuperação funcional ter sido aplicado em um único momento (por volta de dois anos após a cirurgia), sem a possibilidade de comparação com a capacidade funcional pré-fratura e, assim, não podendo avaliar o grau de recuperação dos pacientes. Significa que os valores apresentados pelo estudo representam (em porcentagem) a capacidade funcional atual dos pacientes. Por exemplo, o valor obtido pelo ERF de 63,7 em nosso estudo significa que, atualmente, o paciente apresenta 63,7 \% da capacidade funcional máxima.

No seu estudo, Zuckerman et al ${ }^{(10,28)}$ estratificaram os pacientes em cinco grupos, baseado na avaliação do

\section{REFERÊNCIAS}

\footnotetext{
1. Skinner HB. Current - diagnosis \& treatment in orthopedics. 4th ed. Califórnia: Lange Medical Books/McGraw-Hill; 2006. p.152-6.

2. Chang KP, Center JR, Nguyen TV, Eisman JA. Incidence of hip and other osteoporotic fractures in elderly men and women: Dubbo Osteoporosis Epidemiology Study. J Bone Miner Res. 2004;19(4):532-6.

3. Kannus P, Parkkari J, Sievanen H, Heinonen A, Vuori I, Jarvinen M. Epidemiology of hip fractures. Bone. 1996;18(Suppl 1):57-63.

4. Zuckerman JD. Hip fracture. N Engl J Med. 1996;334(23):1519-25.

5. Davenport MG Fractures, Hip.. Department of Emergency Medicine, New York
}

estado pré-fratura. Escores de 90 a 100: 67\% dos pacientes; escores de 80 a 89: 14\%; escores de 70 a 79: 6\%; escores de 60 a 69: 3,7\%; e escores menores do que 60: 8,3\%. Nosso estudo evidenciou tamanhos muito diferentes do estudo de Zuckerman et al ${ }^{(10,28)}$ nos grupos com escore de 90 a 100: 25\% dos pacientes; e com escore menor que 60: 25\%. Isso prejudicou a comparação entre os achados.

No estudo de Zuckerman et al ${ }^{(10,28)}$, o ERF foi significantemente mais baixo três meses após a fratura comparado com o estado pré-fratura ( $p<0,001)$. Houve aumento significante no escore entre três e seis meses $(p<0,001)$ e entre seis e 12 meses ( $p<0,001)$. Estes achados foram compatíveis com achados anteriores da literatura $^{(41,42)}$. Ele demonstrou que a fratura do quadril resultou em aproximadamente $20 \%$ de perda funcional ao estado pré-fratura durante o primeiro ano quando comparado com um grupo controle. Escores de 90 a 100 e 80 a 89 mostraram um padrão paralelo de recuperação da capacidade funcional prévia à fratura, mostrando $82 \mathrm{e}$ $77 \%$, respectivamente. O grupo que apresentou o menor escore pré-fratura $(E R F<60)$ recuperou praticamente $100 \%$ da capacidade funcional. Os grupos com escores médios pré-fratura (60 a 69 e 70 a 79) foram os que menos recuperaram a capacidade funcional prévia à fratura. Isso os identificou como o grupo de risco e que provavelmente teria maior necessidade de intervenção do que os outros grupos.

\section{CONCLUSÃO}

Assim, podemos concluir que a idade de 80 anos ou mais e escore pré-operatório ASA 3 ou 4 podem ser considerados como fatores de risco para predizer mortalidade em dois anos do pós-operatório por fratura do quadril. O escore ASA, isolado, não tem relação com a capacidade funcional a longo prazo. O tempo de espera para a operação não teve relação com a mortalidade no primeiro ano pós-operatório nem com a capacidade funcional a longo prazo.

University/Bellevue Hospital Center. [acesso em 10 nov. 2010]. Disponívem em: http://emedicine.medscape.com/article/825363-overview"

6. Gurr DE, Gibbs MS. Femur and hip. In: Rosen's emergency medicine: Concepts and clinical practice. 5th ed. St. Louis: Mosby; 2002. p. 643-74.

7. Geiderman J. Hip injuries. In: Harwood-Nuss A, Linden $\mathrm{CH}$, editors. The clinical practice of emergency medicine. Philadelphia: Lippincott-Raven Publishers; 1991.p.407-9.

8. Delee JC. Fractures in adults. Lippincott-Raven Publishers; 1996. p. 1659-63.

9. Nakano T. Prognosis and outcomes of hip fractures. Clin Calcium. 2006;16(12):1999-2004. 
10. Zuckerman JD, Koval KJ, Aharonoff GB, Skovron ML. A functional recovery score for elderly hip fracture patients: II. Validity and reliability. J Orthop Trauma. 2000;14(1):26-30.

11. Stenvall M, Olofsson B, Nyberg L, Lundström M, Gustafson Y. Improved performance in activities of daily living and mobility after a multidisciplinary postoperative rehabilitation in older people with femoral neck fracture: a randomized controlled trial with 1-year follow-up. J Rehabil Med. 2007;39(3):232-8.

12. Press Y, Grinshpun Y, Berzak A, Friger M, Clarfield AM. The effect of co-morbidity on the rehabilitation process in elderly patients after hip fracture. Arch Gerontol Geriatr. 2007;45(3):281-94.

13. Lieberman D. Rehabilitation following hip fracture surgery: a comparative study of females and males. Disabil Rehabil. 2004;26(2):85-90.

14. Heruti RJ, Lusky A, Barell V, Ohry A, Adunsky A. Cognitive status at admission: does it affect the rehabilitation outcome of elderly patients with hip fracture? Arch Phys Med Rehabil. 1999;80(4):432-6.

15. Beloosesky Y, Weiss A, Grinblat J, Brill S, Hershkovitz A. Can functional status, after rehabilitation, independently predict long-term mortality of hip-fractured elderly patients? Aging Clin Exp Res. 2004;16(1):44-8.

16. Sund R, Liski A. Quality effects of operative delay on mortality in hip fracture treatment. Qual Saf Health Care. 2005;14(5):371-7.

17. Elliott J, Beringer T, Kee F, Marsh D, Willis C, Stevenson M. Predicting survival after treatment for fracture of the proximal femur and the effect of delays to surgery. J Clin Epidemiol. 2003;56(8):788-95.

18. Symeonidis PD, Clark D. Assessment of malnutrition in hip fracture patients: effects on surgical delay, hospital stay and mortality. Acta Orthop Belg. 2006;72(4):420-7.

19. Franzo A, Francescutti C, Simon G. Risk factors correlated with post-operative mortality for hip fracture surgery in the elderly: a population-based approach. Eur J Epidemiol. 2005;20(12):985-91.

20. Casaletto JA, Gatt R. Post-operative mortality related to waiting time for hip fracture surgery. Injury. 2004;35(2):114-20.

21. Hakkinen A, Heinonen M, Kautiainen H, Huusko T, Sulkava R, Karppi P. Effect of cognitive impairment on basic activities of daily living in hip fracture patients: a 1-year follow-up. Aging Clin Exp Res. 2007;19(2):139-44.

22. Beloosesky Y, Grinblat J, Epelboym B, Weiss A, Grosman B, Hendel D. Functional gain of hip fracture patients in different cognitive and functional groups. Clin Rehabil. 2002;16(3):321-8.

23. Soderqvist A, Miedel R, Ponzer S, Tidermark J. The influence of cognitive function on outcome after a hip fracture. J Bone Joint Surg Am. 2006;88 (10):2115-23.

24. Beringer TR, Clarke J, Elliott JR, Marsh DR, Heyburn G, Steele IC. Outcome following proximal femoral fracture in Northern Ireland. Ulster Med $\mathrm{J}$. 2006;75(3):200-6.

25. Hasegawa Y, Suzuki S, Wingstrand H. Risk of mortality following hip fracture in Japan. J Orthop Sci. 2007;12(2):113-7.
26. Dzupa V, Bartonicek J, Skala-Rosenbaum J, Prikazsky V. Mortality in patients with proximal femoral fractures during the first year after the injury. Acta Chir Orthop Traumatol Cech. 2002;69(1):39-44.

27. Michel JP, Klopfenstein C, Hoffmeyer P, Stern R, Grab B. Hip fracture surgery: is the pre-operative American Society of Anesthesiologists (ASA) score a predictor of functional outcome? Aging Clin Exp Res. 2002;14(5):389-94.

28. Zuckerman JD, Koval Kenneth J, Aharonoff Gina B, Hiebert R, Skovron M L. A Functional Recovery Score for Elderly Hip Fracture Patients: I. Development. J OrthopTrauma. 2000;14(1):20-5.

29. Bergeron E, Lavoie A, Moore L, Bamvita Jm, Ratte S, Gravel C, Clas D. IS the delay to surgery for isolated hip fracture predictive of outcome in efficient systems? J Trauma. 2006;60(4):753-7.

30. Petrella RJ, Overend T, Chesworth B. FIM after hip fracture: is telephone administration valid and sensitive to change? Am J Phys Med Rehabil. 2002;81(9):639-44.

31. Berger SV, Almeida M, Rosito GA, Polanczyk CA. Pré-operatório. In: Stefani $\mathrm{SD}$, Barros E, editores. Clínica médica: consulta rápida. 2a. ed. Porto Alegre: Artmed; 2002. p. 335-47.

32. Morgan JR, Mikhail MS, Murray MI, Larson CP Jr. Cinical anesthesiology. 3rd ed. New York: Lange-McGraw-Hill; 2002. p. 1-14.

33. Richmond J, Aharonoff GB, Zuckerman JD, Koval KJ. Mortality risk after hip fracture. J Orthop Trauma. 2003;17(1):53-6.

34. Hamlet WP, Lieberman JR, Freedman EL, Dorey FI, Fletcher A, Johnson EE. Influence of health status and the timing of surgery on mortality in hip fracture patients. Am J Orthop. 1997;26(9):621-7.

35. McGuire KJ, Bernstein J, Polsky D, Silber JH. The 2004 Marshall Urist award: delays until surgery after hip fracture increases mortality. Clin Orthop Relat Res. 2004;(428):294-301.

36. McLeod K, Brodie MP, Fahey PP, Gray RA. Long-term survival of surgically treated hip fracture in an Australian regional hospital. Anaesth Intensive Care. 2005;33(6):749-55.

37. Williams A, Jester R. Delayed surgical fixation of fractured hips in older people: impact on mortality. J Adv Nurs. 2005;52(1):63-9.

38. Bergner MB, Bobbitt RA, Carter WB, Gilson BS. The SIP: development and final revision of a health status measure. Med Care. 1981;19(8):787-805.

39. Guyatt G, Walter S, Norman G. Measuring change over time: assessing the usefulness of evaluative instruments. J Chronic Dis. 1987;40(2):171-8.

40. Ware JE. SF-36 Health Survey. Manual and Interpretation Guide. Boston: Nimrod Press; 1993.

41. Magaziner J, Simonisick EM, Kashner TM, Hebel JR, Kenzora JE. Predictors of functional recovery one year following hospital discharge for hip fracture: a prospective study. J Gerontology 1990;45(3):101-17.

42. Mossey JM, Mutran E, Knott K, Craik R. Determinants of recovery 12 months after hip fracture: the importance of psychosocial factors. Am J Public Health $1989 ; 79(3): 279-86$ 